\title{
Nursing, Psychological Intervention and Self-Management after Deep Brain Stimulation in Parkinson's Disease
}

\author{
Lizhen Wu, Yajuan Ke, Yunwan Chen, Fangtan Xing, Yanman Chen \\ Hainan General Hospital, Haikou, China \\ Email: g2002m@163.com
}

How to cite this paper: $\mathrm{Wu}, \mathrm{L} . \mathrm{Z} ., \mathrm{Ke}, \mathrm{Y} . J$. , Chen, Y.W., Xing, F.T. and Chen, Y.M. (2020) Nursing, Psychological Intervention and Self-Management after Deep Brain Stimulation in Parkinson's Disease. Journal of Behavioral and Brain Science, 10, 517-530. https://doi.org/10.4236/jbbs.2020.1012031

Received: November 1, 2020

Accepted: December 4, 2020

Published: December 7, 2020

Copyright $\odot 2020$ by author(s) and Scientific Research Publishing Inc. This work is licensed under the Creative Commons Attribution International License (CC BY 4.0).

http://creativecommons.org/licenses/by/4.0/

(c) (i) Open Access

\begin{abstract}
Parkinson's disease (PD) is a neurodegenerative disease mainly caused by motor disorders, mostly occurring in middle-aged and elderly people. The incidence of PD has been increasing year by year, and up to now, PD is still an incurable disease. However, more and more data show that early implementation of deep brain stimulation and early medical, psychological, social and other interventions can significantly improve the quality of life and prolong the survival time of patients with Parkinson's disease (PD). Mental health guidance, cognitive behavioral intervention, psychogenic therapy and scientific nursing for PD patients may improve the functional recovery after Deep Brain Stimulation (DBS) for Parkinson's disease. This paper discusses the nursing and psychological intervention methods of deep brain stimulation (DBS) implantation in patients with Parkinson's disease (PD), aiming to scientifically discuss the clinical effect of nursing psychological intervention and improve the quality of life in patients with Parkinson's disease. Basic nursing and psychological cognitive behavior intervention measures for PD patients can improve the daily activity ability of PD patients, improve the outcome of PD patients, and effectively improve the satisfaction of PD patients with nursing work, which has certain clinical promotion significance.
\end{abstract}

\section{Keywords}

Parkinson's Disease, Deep Brain Stimulation, Nursing, Psychological Intervention

\section{Introduction}

After Alzheimer's disease, Parkinson's disease (PD) is the second most severe progressive neurodegenerative disease affecting the elderly in the world, with 
static tremor, myotonia, motor retardation and abnormal postural gait as the main clinical motor symptoms [1] [2]. It is considered to be the most serious movement disorder in the world, with a very high disability rate [3]. In China, the prevalence rate among people over 65 years old is 170/100,000 and increases with age, bringing heavy burden to families and society [4]. The main pathological changes of the disease in the substantia nigra compacta dopaminergic neuron loss and Louis corpuscle forming, its main biochemical changes in the striatum region reduced dopamine neurotransmitter, clinical symptoms, including static tremor, muscle rigidity, bradykinesia and posture balance disorder symptoms [5] and hyposmia, fast phase rem sleep behavior abnormalities, the movement symptoms such as constipation and psychological barriers [6]. Frozen gait and flustered gait are the most common types of gait disorders in Pd patients, which seriously affect the ability and quality of life of the patients. Deep brain stimulation (DBS) is currently recognized as the preferred surgical method for adjuvant Parkinson's disease drug therapy at home and abroad [7]. When the symptoms cannot be controlled after repeated dosing, many patients begin to choose the mature DBS surgical treatment, and achieve better therapeutic effect through electrode stimulation and drug therapy after surgery. However, long-term gait changes have become a major obstacle to the improvement of postoperative symptoms in many patients, so how to make patients recover faster gait and improve their quality of life has been a research hotspot. Self-management efficacy is the core concept in the self-management theory. Based on the self-efficacy theory proposed by The American scholar Bandura, it refers to the confidence of individuals in their successful implementation of specific self-management behaviors [8]. Research on self-management efficacy of Parkinson's disease is in its infancy in China, and there are even fewer studies on patients after DBS. Studies have shown that the self management efficiency and the movement of patients with confidence are closely related to overcome difficulties, so this paper aims to DBS patients with postoperative nursing, psychological intervention and self management efficiency and gait rehabilitation status and quality of life to guide, and to explore the perioperative nursing and psychological intervention method, in order to further improve the ability of self-management in patients with, to help accelerate the process of gait rehabilitation patients, eventually improve the quality of life.

\section{Surgical Methods and Medication Guidance}

Over the past few decades, deep brain stimulation (DBS), with its superior efficacy and fewer complications, has been gradually replacing the traditional stereotactic ablation in the field of functional neurosurgery. Target coordinates were obtained through head MRI volumetric scan using THE CRW stereo orientation system of Radionics. Microelectrodes with a tip diameter of about 20 - 30 um were used during the operation, and electrophysiological records were made from $10 \mathrm{~mm}$ on the target. Target positions were selected as Subthalamic 
nucleus (STN), and implanted as deep brain electrodes after the target was confirmed by electrophysiological records [9]. The symptom improvement rate of PATIENTS with DBS is quite ideal, and DBS has the advantages of non-permanent nerve damage and readjust stimulus parameters at any time, which greatly reduces the occurrence of postoperative complications. DBS surgery was initially applied to diseases of motor dysfunction, and several targets [10] were often located in the Subthalamic nucleus, ventral intermediate nucleus of thalamus, and medial part of globus pallidus. In recent years, DBS has expanded into other fields such as epilepsy, essential tremor, depression and intractable pain. The mechanism of ACTION of DBS is quite complex, including inhibition of neuronal discharge, neurotransmitter consumption, excitation or blocking of inhibitory nerve loop and functional block, and damage effect in and around the stimulation of nuclear mass. There is ample evidence [11] that DBS improves the motor symptoms of Parkinson's tremor, muscle stiffness, and motor retardation.

Guidance of medication in clinical treatment: there are many drugs commonly used in Parkinson's patients, which are taken daily. Some newly diagnosed Parkinson's disease patients are not familiar with medication rules, with average effect but significant drug side effects. Therefore, nursing staff should give correct medication guidance to standardize patients. Take medicine in accordance with the doctor's advice, inform the patient of the effect of drug treatment, review regularly, and increase the patient's compliance with taking medicine; Inform patients that taking anti-Parkinson's drugs while eating other foods can have an impact on the body; Proteins in certain foods interfere with the absorption and transport of 1-dopamine, thus affecting the efficacy. Therefore, instructing patients to take medicine one hour before or after meals is helpful to increase the utilization rate of medicine and improve the curative effect.

\section{Care}

\subsection{Preoperative Nursing}

With the gradual increase of PD patients, many scholars have studied the psychological and emotional states of PD stimulation electrode implantation. The technique of stimulating electrode implantation is a safe and effective treatment method, but after all, it produces psychological stress on the patient, and the patient has little knowledge about this aspect, which will produce a strong stress response after the treatment. Domestic and abroad a large number of studies have shown that in patients undergoing surgical treatment there is a widespread stress reaction (such as cancer patients), have given priority to with anxiety psychological stress, anxiety and other negative emotions vagus nerve excitability enhancement, causes the body to promote materials such as catecholamine release, cause the negative emotions such as anxiety and major depression, if not timely intervention, the treatment after the effect is not ideal value. Before, during and after PD stimulation electrode implantation, it is very necessary to take 
certain psychological intervention measures for the patients to improve their adaptability and coping ability and reduce the generation of negative emotions such as anxiety. Parkinson's disease is a slow process, early disease, patients have a certain ability to work, the life can provide for oneself, with the progress of the disease, the gradual loss of labor ability, a significant reduction in the ability to care for life, more patients with anxiety, depression and other emotional, combined with the patients hospitalized with worry about DBS the effect after treatment with DBS high treatment costs can be proportional, patients with anxiety. Talk to patients differently according to their personality characteristics, education level and existing psychological problems [12]. And establish a good relationship between nurses and patients, patients to actively communicate with the patient, understand the psychological status of patients, the patients on the need for surgery, surgical principle, operation method, preoperative postoperative self-care matters needing attention, introduced after the operation, to communicate with patients, eliminate their anxiety and nervousness, actively cooperate with treatment.

\subsection{Preoperative Nutritional Support}

The main symptoms of Parkinson's disease are tremor and rigidity, and the body's energy expenditure increases relatively. Guide patients to eat enough calories, sugar and protein according to patients' age, symptoms and activity level. Food should be light and less salt. Easy to digest, easy to chew and swallow. No alcohol and stimulating food, such as coffee, chili, mustard, curry, etc. Patients taking dopamine therapy limit protein intake, so as not to affect the absorption of dopamine, reducing the efficacy.

\subsection{Nursing Measures for Constipation}

Constipation is very common in Parkinson's disease patients, with an incidence of about $73.0 \%$ reported abroad [13]. The reason is that most of the patients are elderly, with less activity, decreased gastrointestinal function, slow bowel motility, and less drinking water. The nurse should instruct the patient to massage the abdomen twice a day, clockwise for 5 minutes and counterclockwise for $5 \mathrm{mi}$ nutes each time, with the technique changing from light to heavy. Develop the habit of regular daily defecation, guide patients to eat more fresh vegetables and fruits, so as to provide a variety of vitamins, dietary fiber to promote bowel motility and prevent constipation. To perspire much patient, pay attention to compensatory water.

\subsection{Safety Measures and Life Care}

Parkinson's patients have different degree of muscle tension increased, lead to different degrees of bradykinesia, muscle stiffness posture gait abnormalities, memory and ability to respond to all have varying degrees of decline [14], the proper attention to the problem of the safety of the patients, patients admitted to 
hospital after a nurse put patients reach the thermos bottle on the table of the head of a bed, and told the patient not to water into water. Wear non-slip shoes when walking and use a cane or walker. If the patient has difficulty in squatting and standing up, he/she can sit on a high stool to defecate. To the life of joint stiffness cannot take care of oneself patient, should undertake to its daily passive limb activity and muscle, joint massage, in order to promote the blood circulation of limb. For those who cannot eat, they need someone to feed them, to wear and take off clothes, to button up, to tie belts and so on. Take good morning and evening care for bedridden patients, turn over on time, buckle back, take good care of lungs and skin, prevent the occurrence of falling pneumonia and bedsore.

\subsection{Postoperative Nursing}

The most important care measure for Parkinson's disease is to provide safe care to prevent the patient from falling down and being injured. Can be placed on both sides of the stairs or on the wall support bars, especially in the wall near the door handle armrest Settings, improve the safety of patients open, close the door. Handrails are installed in toilets, bathrooms and other places, which can effectively prevent patients from accidentally falling down when putting on and taking off clothes or relieving themselves. Patients with severe tremor should be placed in an armrest chair. The second is to ensure the level of the ground, carpet without wrinkles, so as not to trip patients, when necessary can use a cane to assist. A bed bar is set beside the patient's bed, and a rope is tied at the end of the bed, so that the patient can sit up with the rope, improving the safety. If the home has the sharp Angle must carry on the processing to it, simultaneously forbids the patient to use the sharp instrument, the dangerous goods and so on May cause the influence to the patient the article. Patients with cognitive impairment should be assigned to accompany them. Secondly, patients are encouraged to drink more water, eat more food such as high fiber, sesame oil and honey, and regularly massage the abdomen to develop a good habit of regular defecation. When medication is needed, laxatives can be given according to the doctor's advice. Finally, the patient's clothing is to be as light and loose as possible, the greatest degree to reduce the stimulation of the skin, friction. For bedridden patients should use air mattress, or with soft and breathable thick bedding instead. Keep the sheets smooth and dry. At least 1 to 2 times a day with warm water skin cleaning, changing clothes should be timely.

\subsubsection{Postoperative General Nursing}

Routine nursing after general anesthesia, rest in bed within 24 hours, reduce activities, closely observe whether there is active bleeding, as well as changes in vital signs, pupils, wound dressing and subcutaneous conditions, body movements, language and swallowing function, and report to the doctor in time if there is any problem. Hemostatic drugs are routinely administered to prevent intracranial hemorrhage, and antibiotics are also applied to prevent infection. To prevent edema of brain tissue, oxygen uptake was continuously low $6 \mathrm{~h}$ after op- 
eration. After $6 \mathrm{~h}$ of fasting, according to the patient's chewing ability and swallowing function, the patient should choose a suitable diet, pay attention to food collocation, improve the patient's appetite, and ensure the nutritional intake.

\subsubsection{Surgical Incision Care}

Keep the environment of the hospital room clean and the temperature is appropriate. Change the headrest towel once a day and the hospital gown every day within three days after the operation, and keep the local skin clean and dry. Avoid local skin scratching, resulting in local rupture, infection. Close observation of the incision site infiltration fluid situation. Compression dressing of thorax and abdomen incision is to prevent subcutaneous bleeding and effusion. Patients with chest incision should avoid excessive pulling when moving the operative limb.

\subsubsection{Observation and Nursing of Complications}

1) Complications related to surgery: 15 patients in this group presented $1-3$ days of continuous somnolence, euphoria, irritability, increased speech and other mental symptoms after surgery. Among them, drowsiness was caused by excessive cerebrospinal fluid loss and intracranial gas accumulation caused by surgery. After the elimination of intracranial hemorrhage by CT examination, explain to the family members to eliminate concerns, and give intermittent low-flow oxygen inhalation as prescribed by the doctor. Nurses should make intensive rounds and wake patients at least once an hour to observe changes in consciousness. Assist the patient to eat once every $4-6 \mathrm{~h}$ to ensure the necessary postoperative nutrition; Put the caller at the patient's hand and ask the patient not to walk on his own, but to be supported by the nurse. Other psychiatric symptoms are caused by intracranial gas accumulation in the patient, presenting as restlessness, multilingualism, day and night wakefulness, etc. The nurse will put on extra bed to protect the patient, give protective restraint as prescribed by the doctor, and give sedative drugs when necessary. The symptoms disappeared 7 - 10 days after the operation. 2) Stimulation-related complications, also known as abnormal movement, should pay attention to observe the abnormal movement of the limbs, timely notify the doctor to adjust the parameters of the stimulator, timely add bed covers for the bedridden patients, the bed covers are wrapped with soft cloth, patients should be taken care of when they get out of bed, to prevent falls; This group of patients have one or more of the following symptoms during boot test: transient limb numbness, limb or mouth twitching, dizziness, palpitations, speech influence, staring at the same direction, etc. It is also crucial for nursing staff to observe the improvement of patients' symptoms while observing the above symptoms during boot debugging.

\subsubsection{Functional Exercise}

After the deep brain electrical stimulation, it is generally about four weeks of boot debugging, so the postoperative symptoms of the patients are not obvious, should be gradually after the limb function exercise in independent activity. 
During the period of 1 to 3 days after the operation, start from the bed training, 3 - 5 times per day, 15 - 20 minutes per time, with the limbs moving freely on the bed, gradually moving from small joints to large joints [15]. Nurses should do passive exercise for patients with joint stiffness, and wear the anti-thrombotic pressure belt for patients according to the doctor's advice to prevent the formation of venous thrombosis. Three days after the operation, the patient was encouraged to sit up in bed first. After no discomfort, the patient was accompanied by the nurse to get out of bed for exercise, gradually increasing the number of exercises and extending the exercise time. No strenuous exercise was allowed to prevent falling down. The third to seventh day after the operation is the high incidence of edema around the lesion. When the edema is serious, the muscle strength of one side of the limb will decrease and there will be a deviation in walking. When the above situation occurs, explain the reason to the patient and assist in walking, and pay attention to the intensity and amplitude of exercise step by step. The focus of nursing is to guide the overall coordination of the limbs. After functional exercise, the muscle strength of the group was basically restored to normal at the time of discharge and the group was able to take care of itself.

\subsection{Discharge Guidance and Health Education}

\subsubsection{Discharge Guidance}

The stitches were removed one week after the operation, and the dressing was kept for 3 days. A week later, the patient could take a bath to avoid local scratching and infection. Regularly went to our outpatient department for debugging and guided the patients and their families to observe the improvement and change of symptoms of Parkinson's disease. In addition, instruct the patient to use the remote-controlled magnet switch correctly, and inform the patient to avoid being hit at the incision in the chest and abdomen, which will cause damage to the battery. No strenuous activity, reduce the friction between the battery and local skin; Stay away from high heat environment, such as sauna room; Keep away from magnetic environment, such as refrigerator, speaker and microwave oven. It is recommended to carry an imprinted IDENTIFICATION card with you when going out for activities so that you can get help when necessary. The battery can be used for 5 - 10 years, and the pulse generator can be replaced surgically if the battery runs out.

\subsubsection{Health Education}

After surgery, levodopa drugs such as madopa or rine should be continued. Adjust the dosage as prescribed by your doctor. Since the operation cannot solve the problem of lack of dopamine in the brain, the operation needs to cooperate with the drug treatment to give full play to its best effect. Meanwhile, the operation can eliminate the side effect of movement fluctuation brought by the drug, so as to make the drug give full play to its best effect. Patients should be informed of the incompatibility of drugs, such as vitamin B6, which will reduce the 
efficacy of mdopa, should not be taken together; Patients should eat less food with high cholesterol, because the amino acids in the diet will affect the absorption of madopa in the small intestine, making it less effective, etc. In patients treated with anticholinergic drugs, simultaneous consumption of foods rich in cholinergic drugs may reduce the efficacy of anticholinergic drugs, or may aggravate the disease. Let Parkinson's patients and their families more fully understand the disease, know the occurrence and development of the disease, so as to cooperate with the treatment. When attending the hospital, the responsible nurse makes a comprehensive assessment of the patient in all aspects, such as name, gender, age, contact information, hobbies, living habits and other personal information, and establishes personal records to provide reference basis for the diagnosis, treatment and prognosis of the patient; Tell the patient how to use the medicine correctly, stick to the medicine and how to deal with adverse drug reactions. Pay attention to the temperature and humidity in the patient's room to prevent the upper respiratory tract infection caused by cold; Adhere to participate in the right amount of activities and physical exercise, exercise should be based on the condition and physical fitness, grasp the way, intensity and time, so as not to exercise too much and aggravate the condition; Good skin care work; Life should be regular, to ensure adequate rest and sleep, conducive to the recovery of physical; Rationalization of diet and nutritional structure helps to improve the condition.

\section{Psychological Nursing}

Psychological stress of patients includes physiological and psychological changes, the former is reflected in the endocrine system changes and the resulting changes in the immune system; The latter is embodied in the emotional experience dominated by anxiety and fear and the explicit behavior caused thereby. Foreign studies have shown that the degree and duration of stress are related to the level of anxiety, and it is believed that the fear of patients before treatment can be reduced and the recovery after treatment can be affected by anxiety through behavioral and physiological mechanisms. Pretreatment anxiety had less to do with the severity of the disease and more to do with personality and coping style. Coping is an important mediating factor in the process of stress. The response of patients after treatment varies according to the different coping styles before treatment. As a strong source of psychological stress, deep brain stimulation surgery for Parkinson's disease, in addition, intraoperative local anesthesia (craniotomy under consciousness) will cause patients to produce a psychological stress response dominated by anxiety. Anxiety is an unpleasant emotional experience characterized by nervousness, fear, worry and neuroticism, accompanied by hyperfunction of the botanic nerves. It is the frustration feeling when one anticipates the threat of uncertain internal and external stimuli and feels powerless to cope with it. This kind of complex emotion has both positive and negative effects on stress response. Certain anxiety helps to improve the 
body's psychological tension and enhance its adaptability to stress sources, but too much anxiety weakens this ability. Coping refers to people's continuous efforts to manage the changing process of psychological stress from cognition and behavior. When the situation can be changed, the patient will adopt a coping style based on problem-solving, and when the situation cannot be changed, the patient will adopt a coping style based on emotional adjustment. Individual coping style has great influence on the nature and intensity of stress response. As a mediating and regulating mechanism of stress and health, coping plays a protective role on physical and mental health. It is generally believed that facing is a positive coping style, avoiding and yielding is a negative coping style, and a mature coping style is helpful to reduce the level of anxiety. To this disease patient, want to undertake encouraging to its more, speak oneself feeling to wait, give psychological support timely. Help patients to actively participate in social activities and participate in activities that patients are interested in, to carry out the cultivation of interest in life, can be more such as outing, travel and other activities, which can not only make patients excited, but also help patients to alleviate the state of rigidity. Although the disease can not be a radical cure of the situation to inform the patient and his family as soon as possible, let its psychological preparation, at the same time to its comfort, although not a radical cure but after drug treatment can reduce the symptoms, increase the patient and his family in the face of the disease confidence, mobilize the enthusiasm of patients, encourage patients to take the initiative to cooperate with treatment.

\subsection{Psychological Guidance}

Elderly patients often refuse to hospital, the hospital for most of Parkinson's disease, there are more or less negative guidance and psychological shadow, especially for older patients, nursing staff should be timely to correct psychological counseling [16], guide the patients to avoid overly emotional tension in the course of the disease, positive and optimistic; at the same time, successful rehabilitation cases can be used to guide patients to build confidence and actively cooperate with treatment, so as to achieve good curative effects and improve the quality of life of patients. Patients are encouraged to get in touch with people and things in society and participate in recreational activities of the elderly.

\subsection{Psychotherapy}

Individualized psychological therapy is adopted according to different people [3]. Treatment before the preliminary understanding of the patient's family background, education, social events, personality traits, such as information, the wall of the language, behavior, mental activities, and then through effective communication with patients, understand their inner thoughts, find out the possible methods to treatment in patients with doubt, patiently for patients and their families, guide them cooperate with treatment. When the patient is agitated, fear and other unstable emotions, it is necessary to communicate with him 
in a timely manner, and pay attention to the speed and tone of speech during communication. Firstly, it is necessary to understand the factors of the patient's fear, and then use the surrounding things to divert the patient's attention, such as doing some exercise.

Exercise, read novels or tell jokes, etc. to help patients stabilize their emotions and promote therapeutic effect. Or in a bad mood when patients, often feel pessimism and despair, to take the initiative to give the care of relatives, share the patients' life, discuss the patient's hobby, highlight the patient a bit and achievements, to give praise and encouragement, necessary multidirectional guide patients they tell their ideas, say goodbye to the lonely, win the affirmation, strengthen to yearn for and confidence in life. When the patient's sleep quality is not good or wake up in the sleep, timely comfort, and tell the patient some self-sleep methods, such as reading, listening to some elegant music or silently counting Numbers.

\subsubsection{Behavioral Therapy}

Progressive muscle relaxation is a behavioral therapy to help patients under psychological stress to carry out relaxation training. Through conscious tension and relaxation of muscle groups in all parts of the body, the tension is relieved from the top to the bottom of the body and the relaxation is achieved step by step, so as to control and relieve stress response and promote the harmony of body and mind. Explain the procedure, purpose and precautions of the exercise in detail to the patients and their families, and use clear, clear, understandable and gentle instructions to help guide the patients to master the main points in the form of action demonstration. Keep the training environment quiet, clean and with soft lighting. Instruct patients with natural and comfortable to lie on your back in bed, follow the instructions say "relax" and "tension", from top to bottom have consciously in order to relax muscles tense before exercise, to the whole body muscle relaxation and bed lie down, feeling relaxed and nervous when different experience of body and mind, and on the basis of this silence, relaxed, soft music, help patients slowly into the subconscious intentional imagination, ease tension in beautiful pictures, to assist the coordination of body and mind, unity, peace, improve physical and mental pleasure, reduce anxiety tension stress state, etc. Starting from the day of appointment, each relaxation technique should be practiced $0.5 \mathrm{~h}$ before the patient goes to bed. Starting from the day of appointment, the relaxation technique should be practiced once a day for a total of 2 times, 15 - 25 minutes each time, to avoid interruption and interruption during the whole practice process.

\subsubsection{Music Therapy}

Music therapy refers to a treatment method in which patients enjoy some music, or they sing and play to themselves to maintain a good mood and thereby obtain or promote the progress of treatment. Shakespeare once said that "music is the food of the soul next to love". It is the product of the integration of medical 
psychology and music proposed by scholars. The data showed that people with Parkinson's disease were happier, had more daily activities and were significantly more emotionally stable after music therapy.

\section{Other Care}

\subsection{Nutrition Care}

Ensuring good nutrition and physical condition is important in improving the health of people with Parkinson's disease. By adjusting the diet structure, the absorption of drugs achieves the best therapeutic effect. The diet of Parkinson's disease patients needs to be adjusted according to their own conditions: food variety, happy eating, safe eating environment, so that patients can enjoy eating; Limit meat intake, often eating moderate amounts of fresh milk and soy; Eat less fatty meats, lard and animal offal; Avoid bone spurs and indigestible foods; Drink six to eight glasses of water a day. In order to maintain the repair of some damaged nerve cells, 400 micrograms of folic acid should be supplemented daily [17]. Nursing a patient with Parkinson's disease should be evaluated before eating, and the patient's swallowing reflex, oral movement control, cough, vomiting reflex, and swallowing ability should be evaluated in advance. And help the patient to sit for 10 - 15 minutes after the meal. If the tremor is mild and the chopsticks are difficult to hold, use a fork with a large handle, a spoon, and a non-breakable stainless steel rice bowl or water cup. If the patient's fingers tremble severely, the bowl, spoon, etc. can be temporarily fixed on his hand, if necessary to help him eat. Due to the special condition of the patient, the medical staff should not urge the patient to eat, but the food temperature should be maintained, in order to avoid the patient to eat too hot, too cold food. For food choice, to easy to chew, easy to digest, easy to swallow food as the standard, can be chopped food or give semi-fluid, so convenient for the patient chew, swallow. Nasal feeding should be performed for patients who are prone to cough [18]. When necessary, nutritional supplements can be supplemented by intravenous supplementation. Since a high-protein diet can reduce the efficacy of levodopa, patients should not blindly eat too much protein. If possible, the patient can be provided with a relaxed and pleasant eating environment and sufficient time for eating. What's more, it is necessary to observe and record the improvement of patients' nutritional status and weight changes.

\subsection{Rehabilitation Training}

Appropriate rehabilitation training has positive significance for early Parkinson's disease patients. The functional training of daily life includes encouraging patients to put on and take off clothes by themselves, and strengthening the coordination training of upper and lower limbs. For those who have difficulty in getting up by themselves, the head of the bed can be raised, and a rope can be tied at the end of the bed to facilitate patients to pull out of bed, so as to avoid sitting on soft sofas and deep sunken chairs. The gills workout involves first 
pressing the gills

Then draw as many of your cheeks as you can, toot and whistle as you can, smile, pout, whistle and puff your face in the mirror. Language training includes helping people with Parkinson's disease with planned pronunciation, reading aloud, and singing.

\subsection{Family Support}

Family encouragement and support is an important part of Parkinson's treatment. Family members' support and encouragement for patients with Parkinson's disease is of positive significance in improving the quality of life of patients, so relatives and friends should be encouraged to give patients enough care and build up their confidence.

\subsection{Emotional Care}

The medical staff must understand that the elderly are already incapacitated, and it is more difficult for them to take care of themselves after the illness. They need help for many conveniences in their daily life. Young people are energetic, quick in thinking and quick in speaking and doing things, but it is difficult for elderly patients to keep up with young people's thinking, which often results in patients' nervous mood. Therefore, medical staff should first look at the problem from the perspective of the patient, be more considerate of the patient's situation, be patient with the patient, give the patient sufficient response time, especially when talking with the patient, medical staff should listen attentively, otherwise the patient will easily feel indifferent. Medical staff should take care of patients with depression with the care of their relatives and should not abandon their idea. Meanwhile, they should teach the family members or caregivers of patients to do the same. Elderly patients should be respected and enjoy the right of medical care. During therapy, understanding conversation is most effective.

When a patient confesses his work, situation or family troubles to the medical staff, we express our sympathy and understanding to the patient through a variety of body language, so that the patient can feel the most genuine care and be willing to talk with the medical staff, so as to maintain a good hospital attitude [19].

\section{Summary and Prospect}

For Parkinson's patients in addition to the general care and health guidance to their life after discharge, first of all, to guide the life of patients with Parkinson's patients avoid emotional in our daily life, stick to attend some appropriate, its activities and physical exercise, as far as possible to the greatest degree of joint activities, prevent secondary joint stiffness. Make sure you get enough sleep and eat a nutritious diet to prevent colds. Psychological counseling is as important as health guidance. Based on the trusting nurse-patient relationship, it communicates with patients, encourages them to express their own ideas and opinions, 
and promotes the contact between patients and the society [20]. Instruct and help patients to keep their clothes clean and their self-image as perfect as possible, improve their ability of self-care and self-care, and enhance their confidence in treatment and life. When the patient needs to take the medicine, the patient must be instructed to take the medicine correctly and insist on taking the medicine according to the doctor's advice. It is better to go back to the hospital regularly for the review of liver and kidney function, and monitor the change of blood pressure at any time. Psychological nursing combined with health education has a good effect on the treatment of PD patients. Combined with clinical practice operation, psychological nursing combined with health education can effectively eliminate patients' fear and tension of operation and prognosis, which can not only promote physical rehabilitation, but also effectively promote psychological and spiritual rehabilitation, thus ensuring patients' all-round health. In addition, psychological nursing combined with health education can effectively promote mutual understanding and support between doctors and patients, and truly meet the physiological and psychological needs of patients, which is worthy of clinical promotion and application.

\section{Foundation Project}

Hainan Health Commission Science and Technology Project (No. 20A200003).

\section{Conflicts of Interest}

The authors declare no conflicts of interest regarding the publication of this paper.

\section{References}

[1] Jia, J.P. and Chen, S.D. (2013) Neurology. People's Medical Publishing House, Beijing, 278

[2] Kalia, L.V. and Lang, A.E. (2015) Parkinson's Disease. Lancet, 386, 896-912. https://doi.org/10.1016/S0140-6736(14)61393-3

[3] Li, H.J., Zhang, M.F., Chen, L., Zhang, J., Pei, Z., Hu, A.L. and Wang, Q. (2010) Nonmotor Symptoms Are Independently Associated with Impaired Health-Related Quality of Life in Chinese Patients with Parkinson's Disease. Movement Disorders, 25, 2740-2746. https://doi.org/10.1002/mds.23368

[4] Zhang, Z.X., Roman, G.C., Hong, Z., Wu, C.-B., Qu, Q.-M., Huang, J.-B., et al. (2005) Parkinson's Disease in China: Prevalence in Beijing, Xi'an, and Shanghai. Lancet, 365, 595-597.

[5] Postama, R.B., Berg, D., Stern, M., Poewe, W., Olanow, C.W., Oertel, W., Obeso, J., et al. (2015) MDS Clinical Diagnostic Criteria for Parkinson's Disease. Movement Disorders, 30, 1591-1601. https://doi.org/10.1002/mds.26424

[6] Chaudhuri, K.R., Healy, D.G. and Schapira, A.H. (2006) Non-Motor Symptoms of Parkinson's Disease: Diagnosis and Management. Lancet Neurology, 5, 235-245. https://doi.org/10.1016/S1474-4422(06)70373-8

[7] Wu, X. and Hu, X.W. (2017) Progress and Prospect of Deep Brain Stimulation in the Treatment of Parkinson's Disease. Chinese Clinical Medicine, 2017, 833-838. 
[8] Bandur, A.A. (1997) Social Learning Theory. Englewood Cliffs Prentice Hall, Upper Saddle River, 79-82.

[9] Zhang, Y.Q., Li Y.J., Zhuang P., et al. (2008) Analysis of the Effect of Deep Brain Stimulation on Non-Motor Symptoms of Parkinson's Disease. Chinese Journal of Microinvasive Neurosurgery, 13, 49-51.

[10] Krack, P., Batir, A., Van Blercom, N., Chabardes, S., Fraix, V., Ardouin, C., et al. (2003) Five Year Follow-Up of Bilateral Stimulation of the Subthala Mic Nucleus in Advanced Parkinson's Disease. The New England Journal of Medicine, 349, 1925-1934. https://doi.org/10.1056/NEJMoa035275

[11] Deuschl, G., Schade-Brittinger, C., Krack, P., Volkmann, J., Schäfer, H., Bötzel, K., et al. (2006) A Randomized Trial of Deep-Brain Stimulation for Parkinson's Disease. The New England Journal of Medicine, 355, 896-908. https://doi.org/10.1056/NEJMoa060281

[12] Wen, C.J., and Jin, X.Y. (2017) Nursing Cooperation of Deep Electrode Implantation in Parkinson's Disease Patients. Nursing and Rehabilitation, 16, 291-293.

[13] Nehra, A. and Moreland, R.B. (2001) Neurogic Erectile Dysfunction. Urologic Clinics of North America, 28, 289-308. https://doi.org/10.1016/S0094-0143(05)70139-7

[14] Martinelly, C.D., Baptiste, P. and Maknoon, M.Y. (2014) An Assessment of the Integration of Nurse Timetable Changes with Operating Room Planning and Scheduling. International Journal of Production Research, 52, 7239-7250. https://doi.org/10.1080/00207543.2014.916827

[15] Zhang, W.J., Wang, H.J. and Zhang, X.H. (2005) Nursing Care of 21 Patients with Tourette's Syndrome Treated by Stereotactic Surgery. Chinese Journal of Nursing, 40, 96-97.

[16] Londer, L.X., Slevin, J.T., Kryscio, R.J., Martin, C.A., Andersen, A.H., Smith, C.D. and Schmitt, F.A. (2013) Dopaminergic Modulation of Memory and Affective Processing in Parkinson Depression. Psychiatry Research, 210, 146-149. https://doi.org/10.1016/j.psychres.2013.06.003

[17] Yu, S.J. and Feng, J. (2014) Analysis of Relationship between Folic Acid Level and Progression of Parkinson's Disease. Journal of China University of Medical Science, 43, 694-697.

[18] Wu, M.Y. (2005) Clinical Nursing of Parkinson's Disease in the Elderly. Anhui Medicine, 9, 303-304.

[19] Huang, J.F. (2004) Identification and Intervention of Depression in Hospitalized Patients. Modern Nursing, 10, 295.

[20] Liao, Y.-L. (2009) Clinical Nursing of 26 Cases of Senile Parkinson's Disease. Qilu Nursing Journal, 15, 63-64. 